Med. thorac. 1965;22:I-III

\title{
Contents, Vol. 22, 1965
}

Internationale Zeitschrift für Erkrankungen der Thoraxorgane

International Review of Thoracic Diseases

Revue internationale des Maladies du Thorax

Cegründet als « Schweizerîsche Zeitschrift für Tuberkulose und Pneumonologie » Vol. 1, 1944 von E. Bachmann, M. Gilbert, F. Häberlin, W. Lðffler, P. Sterner und E. Uehlinger.

\section{REDACTORES}
A. L. d'Abreu, Birmingham
R. H. Behnke, Indianapolis, Ind.
G. Birath, Göteborg
H. Bloch, Basel
G. Brouet, Paris
F. Cardis, Lausanne
R.M. Cherniack, Winnipeg
R.V. Christie, Montreal
G. Daddi, Milano
H. Denolin, Bruxelles
A. B. DuBoiS, Philadelphia, Pa.
E. Dunner, Washington, D. C.
J. Durand, Paris

A. P. Гishman, New York

CM. Fletcher, London

P. Fréour, Bordeaux

H.W. Fritts, Jr., New York

E. A. Gaensler, Boston, Mass.

P. Galy, Lyon

R. Garbagni, Torino

W. Giese, Münster

J. Gough, Cardiff

W. H. Hauss, Münster

J. B. HICKAM, Indianapolis, Ind.

I.T.T.Higgins, Pittsburgh, Pa.

P. Hugh-Jones, London

B. LöHR, Düsseldorf

H. Ludin, Basel

G. R. Meneely, Nashville, Tenn.

R. S. Mitchell, Denver, Colo.

H. L. Motley, Los Angeles, Calif.

A. Omodei Zorini, Roma

N. G. M. Orie, Groningen 
D. D. Reid, London

L. Reid, London

P. H. Rossier, Zurich

P. Sadoul, Nancy

W. Schoedel, Göttingen

W. Steenken, Jr., Saranac Lake, N. Y.

R. E. Steiner, London

C. H. Stuart-Harris, Sheffield

W.T. Ulmeh, Bochum

EDITOR H. Herzog, Basel

Vol. 22

1965

BASEL (Schweiz) S. KARGER NEW YORK

INDEX

Case Reports from the Thoracic Services, Boston University Goff, Anne M. and Gaensler, E. A.:

Primary (Idiopathic) Pulmonary Hypertension 530

Graham, W. G. B. and Gaensler, E. A.:

Talco-Silicosis in a Rubber Worker 590

Pfeffer, Sigrid H.; Desforges, G. and Gaensler, E. A.:

Pulmonary Hemosiderosis and Glomerular Kidney Disease:

Goodpasture's Syndrome 470

Daum, S.: $\quad$ vide Tlustý, L.

Desforges, G.: vide Pfeffer, Sigrid H.

Fréour, P.; Germouty, J. et Puel, B.:

Le devenir fonctionnel des pneumonectomisés $\quad 489$

Gaensler, E. A.: vide Goff, Anne M.; Graham, W. G. B.; Pfeffer, Sigrid H.

Germouty, J.: vide Fréour, P.

Haller, R. de and Reid, L.: Adult Chronic Bronchitis. Morphology, Histochemistry and

Vascularisation of the Bronchial Mucous Glands 549

Hlou§ková, Z.: vide Tlustý, L.

Hofer, P. und Scherrer, M.:

Altersabhängigkeit des alveolo-arteriellen OyPartialdruckgradienten

in Normoxie, Hypoxie und Hyperoxie $\quad 450$

Kennedy, J.H.: Hypoxie Pulmonary Hypertension, Serotonin, and Cardiac Failure:

An Experimental Study 568

Kreukniet, Joh. : Pulmonary Emphysema and Lung Function Studies in Patients with

Chronic-Non-Specific Lung Diseases 433

Kohn, R.: $\quad$ vide Tlustý, L.

Puel, B.: $\quad$ vide Fréour, P.

Rasche, B. und Ulmer, W. T.:

Untersuchungen über die Herkunft der Alveolar- und Peritonealmakro-

phagen (Ein experimenteller Beitrag zum Reinigungsmechanismus der

Lunge) 516

Reid, L.: $\quad$ vide Haller, R. de

Red?, E.: $\quad$ vide Weller, W.

Scherrer, M.: vide Hofer, P. 
Stiksa, J.: $\quad$ vide Tlustý, L.

Tlustý, L.; Hlousková, Z.; Kohn, R.; Daum, S. and Stiksa, J.:

Pulmonary Dififusing Capacity and its Components (Dm and $\mathrm{Vc}$ ) in Children and Young

Persons after Interstitial Pneumonias .... 508

Ulmer, W. T.: vide Rasche, B.

Verhandlungsberichte - Proceedings - Congrès

Proceedings of the Seventh Annual Conference on Research in Emphy

sema, Aspen, Colo., June 10-13, 1964

1

Weller, W. und Reif, E.:

Methode zur Messung absoluter Intrapleuraldrucke mit dem Oeso-

phagus-Ballonkatheter

574

Libhi 480, 546

English Summaries 21, 36, 46, 58, 68, 75, 87, 98,103,116,128,144,156,169,179, 186, 194, 206, 217, 229, 241, 256, 273, 293, 302, 325, 343, 353, 363, 373, 381, 397, 412, 446, 466, 478, 506, $514,528,543,565,571,585,602$ 